\title{
Aspectos biológicos de Stellifer stellifer (Bloch) na pesca artesanal do camarão sete-barbas, Armação do Itapocoroy, Penha, Santa Catarina, Brasil
}

\author{
Laura Ribas de Almeida ${ }^{1}$ \\ Joaquim Olinto Branco ${ }^{1}$
}

\begin{abstract}
Biological aspects of Stellifer stellifer caught in sea-bob-shrimp's artisanal fishery, Armação do Itapocoroy, Penha, Santa Catarina, Brazil. Stellifer stellifer (Bloch, 1790) is one among the most abundant ictiofauna species which are caught by sea-bob-shrimp's artisanal fishery in Armação do Itapocoroy. Aiming to analyze its population structure, a total of 1459 specimens were collected from July/1996 to August/1997. Stellifer stellifer presented lengths varying between 3,7 and $17,7 \mathrm{~cm}$, macroscopic sex differentiation started from $6,0 \mathrm{~cm}$, with a higher frequency of females (sex ratio $=1: 3,2$ ). The relationship between length and weight was $\mathrm{Wt}=$ $0,0073 \mathrm{Lt}^{3,1415}$. First maturation size was determined for females $(7,5 \mathrm{~cm})$ and males $(8,1 \mathrm{~cm})$. The fishery acts in a similar way on juveniles and adults. Stellifer stellifer presented two spawning seasons, one in spring and another in autumn.

KEY WORDS. Stellifer stellifer, biological aspects, artisanal fishery
\end{abstract}

Stellifer stellifer (Bloch, 1790), comumente conhecido como cangoá, canganguá ou cangulo, distribui-se da Venezuela ao litoral sul do Brasil (Santa Catarina), em águas litorâneas e estuarinas com fundos de areia ou lama (MENEZES \& FIGUEIREDO 1980), características estas semelhantes às encontradas nos locais tradicionais da pesca artesanal do camarão sete-barbas Xiphopenaeus kroyeri (Heller, 1862) (BRANCO et al. 1999).

Estudos voltados especificamente às análises populacionais e ecológicas do gênero Stellifer Öken, 1817 restringem-se a S. rastrifer (Jordan, 1889) e S. brasiliensis (Schultz, 1945) no litoral de São Paulo (CoElHo et al. 1985, 1987; GIANNINI \& PAIVA-Filho 1990, 1995); Stellifer rastrifer no Paraná (CHAVES \& VENDEL 1997, 1998) e Santa Catarina (LOPES et al. 1997). Não foram encontradas informações sobre os aspectos biológicos de Stellifer stellifer na bibliografia disponível.

COELHO et al. (1986), citam que apesar da pequena importância econômica ou ausência de interesse, as espécies rejeitadas, pelo menos as numericamente significantes, devem ser estudadas quanto a sua biologia, ressaltando a importância desses conhecimentos para o entendimento do encadeamento trófico das espécies na área de pesca e no equilíbrio dos ecossistemas em explotação.

O presente trabalho tem como objetivo apresentar informações básicas sobre Stellifer stellifer, oriunda da ictiofauna acompanhante da pesca artesanal do camarão sete-barbas realizada na Armação do Itapocoroy, Penha, Santa Catarina.

1) Centro de Ciências Tecnológicas da Terra e do Mar, Universidade do Vale do Itajaí. Caixa Postal 360, 88301-970 Itajaí, Santa Catarina, Brasil. E-mail: branco@cttmar.univali.br; larial2@ hotmail.com 


\section{MATERIAL E MÉTODOS}

As coletas foram realizadas mensalmente durante o período de julho/1996 a

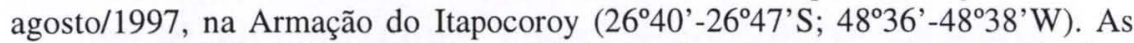
amostragens foram feitas nas áreas tradicionais de pesca do camarão sete-barbas, entre as profundidades de 5,0 a 20,0 m.

Os exemplares foram coletados utilizando-se duas redes-de-arrasto com portas, com malha de $3,0 \mathrm{~cm}$ na manga e no corpo e $2,0 \mathrm{~cm}$ no ensacador, sendo tracionadas por uma baleeira de $10,5 \mathrm{~m}$ de comprimento com motor de $40 \mathrm{Hp}$, com velocidade média de dois nós e duração média de 30 minutos por arrasto. Os exemplares coletados foram etiquetados, acondicionados em sacos plásticos e mantidos em caixas térmicas, para serem processados posteriormente em laboratório.

Paralelamente às coletas dos peixes, foram registrados os dados de temperatura e salinidade da água de superfície. No mês de julho/1997, devido a problemas técnicos não foram registrados esses parâmetros.

No laboratório, os exemplares foram identificados de acordo com MENEZES \& FIGỪEIREDO (1980). A determinação do sexo foi através de observação macroscópico das gônadas. Os exemplares cujo sexo não puderam ser determinado foram agrupados como indeterminados. Foram registrados o comprimento total em $\mathrm{cm}$ (Lt) e o peso total em gramas (Wt). A caracterização macroscópica das gônadas foi realizada de setembro/1996 a agosto/1997, registrando-se o peso e o estádio de maturação (VAZZOLER 1996).

Para verificar a possível diferença entre os sexos durante os meses e por classe de comprimento, foi aplicado o teste $X^{2}$ com nível de significância de $5 \%$ e $\mathrm{n}$-1 graus de liberdade $(\mathrm{n}=2)$. A distribuição da freqüência de comprimento total foi empregada na caracterização sazonal e anual da população. A relação peso-comprimento total foi calculada para os sexos agrupados, de acordo com SANTOS (1978).

O tamanho de primeira maturação $\left(\mathrm{Lt}_{\mathrm{pm}}\right)$ e a época de desova foram ajustados segundo VAZZOLER (1996). Para a análise da atuação da pesca sobre esta população em função da captura de jovens e adultos utilizou-se a distribuição de freqüência por classe de comprimento para os sexos agrupados, baseando-se no tamanho de primeira maturação representado pela média entre machos e fêmeas (L50M).

\section{RESULTADOS}

\section{Temperatura e salinidade}

A temperatura da água de superfície, durante o período de estudo, apresentou uma flutuação sazonal com os maiores valores nos meses de primavera e verão, com máximo de $28,0^{\circ} \mathrm{C}$ registrado em março/1997; os menores ocorreram nas estações de outono e inverno, com valor mínimo $17,0^{\circ} \mathrm{C}$ em julho/1996 (Fig. 1a). A média anual foi de $22,8 \pm 4,0^{\circ} \mathrm{C}$. A salinidade da água de superfície apresentou flutuações ao longo do período de estudo, sendo os maiores teores $(35,0 \mathrm{ppm})$ ocorreram nos meses de outono, e os menores $(30,0 \mathrm{ppm})$ em fevereiro sendo a média anual de $33,4 \pm 1,6$ ppm (Fig. 1b). 

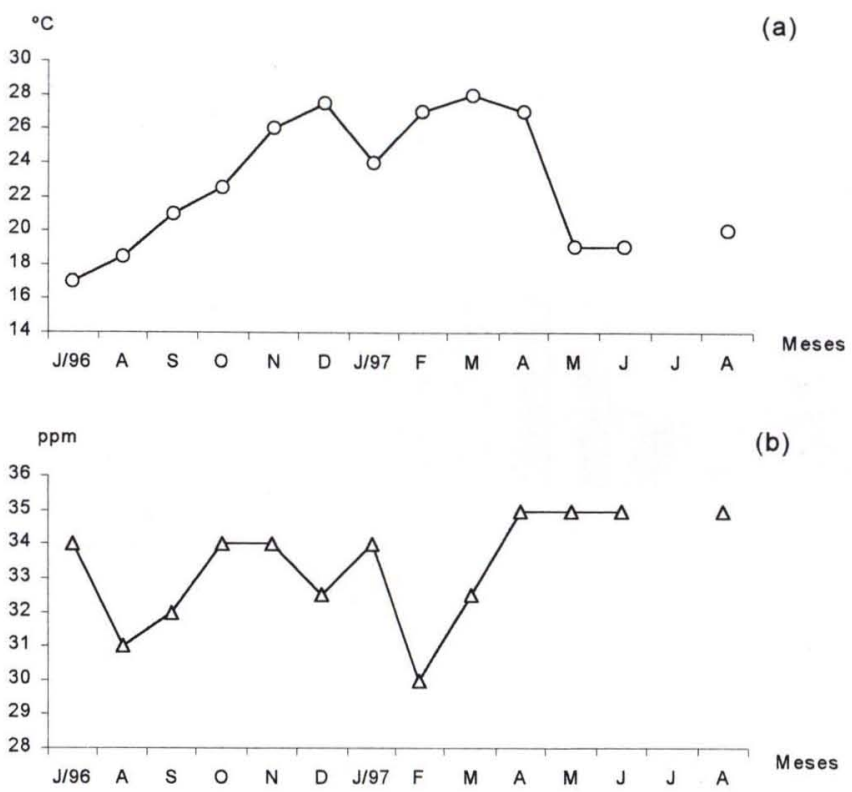

Fig. 1. Variações mensais da temperatura (a) e salinidade (b) da água de superfície.

\section{Estrutura de comprimento}

O número total de $S$. stellifer analisados foi de 1459 exemplares, com média mensal de 104 peixes. A amplitude de comprimento total da população variou entre 3,7 a $17,7 \mathrm{~cm}$, sendo que aproximadamente $75 \%$ dos indivíduos capturados apresentaram comprimento inferior a $11,0 \mathrm{~cm}$, com comprimento médio de $8,3 \mathrm{~cm}$ (Fig. 2).

A determinação macroscópica do sexo foi possível a partir dos $7,0 \mathrm{~cm}$ de comprimento total, sendo que o comprimento máximo para as fêmeas foi de 17,7 $\mathrm{cm}$ e para os machos foi de $16,0 \mathrm{~cm}$ (Fig. 2); as maiores frequiências ocorreram, respectivamente, nas classes de 11,0 e $9,0 \mathrm{~cm}$. Nos exemplares indeterminados a maior freqüência ocorreu na classe de $7,0 \mathrm{~cm}$, decrescendo até os $11,0 \mathrm{~cm}$; entre os comprimentos de $7,0 \mathrm{~cm}$ a $8,0 \mathrm{~cm}$ a separação macroscópica dos sexos não foi muito eficiente (Fig. 2).

Em geral, a ocorrência de exemplares de sexo indeterminados nas amostragens foi relativamente alta, como pode ser visto na tabela I, ultrapassando os $52,0 \%$ de todos os indivíduos coletados. Porém essa captura variou entre os meses, sendo que de outubro/1996 a dezembro/1996 os indeterminados representaram menos de $7,0 \%$ dos exemplares capturados e em setembro/1996 não foi registrada sua ocorrência. Os meses de maior frequiência foram abril/1997, maio e junho, respectivamente com $66,81 \%, 75,45 \%$ e $81,1 \%$ (Tab. I).

Analisando a população independentemente do sexo e do estádio de maturação, verificou-se que na primavera e no outono foram capturados os maiores exemplares (Fig. 3a-c). Na primavera 83,8\% da população esteve representada por indivíduos com comprimento entre 7,0 a $12,0 \mathrm{~cm}$. No verão, a presença dos menores exemplares 


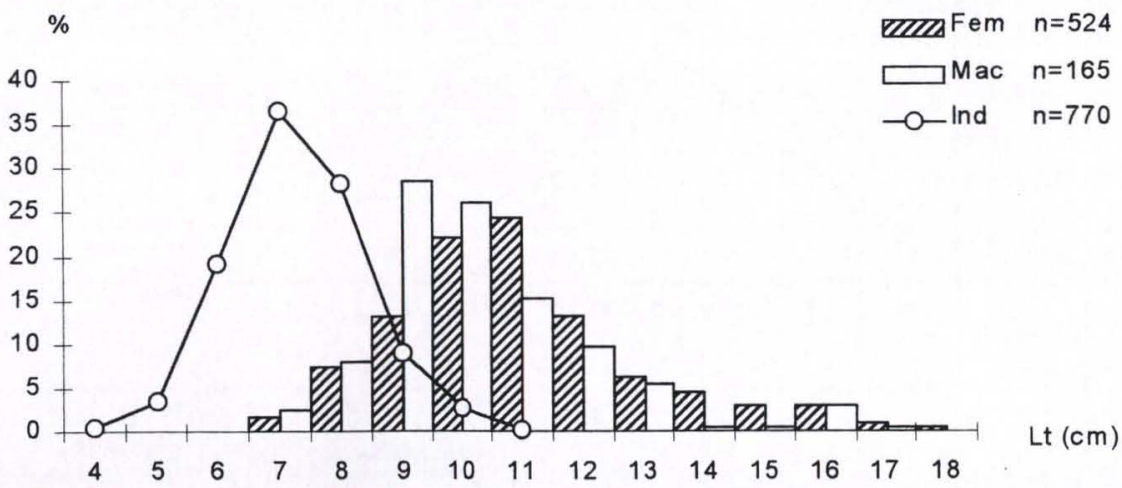

Fig. 2. Freqüência (\%) por classe de comprimento de Stellifer stellifer para fêmeas, machos e indeterminados.
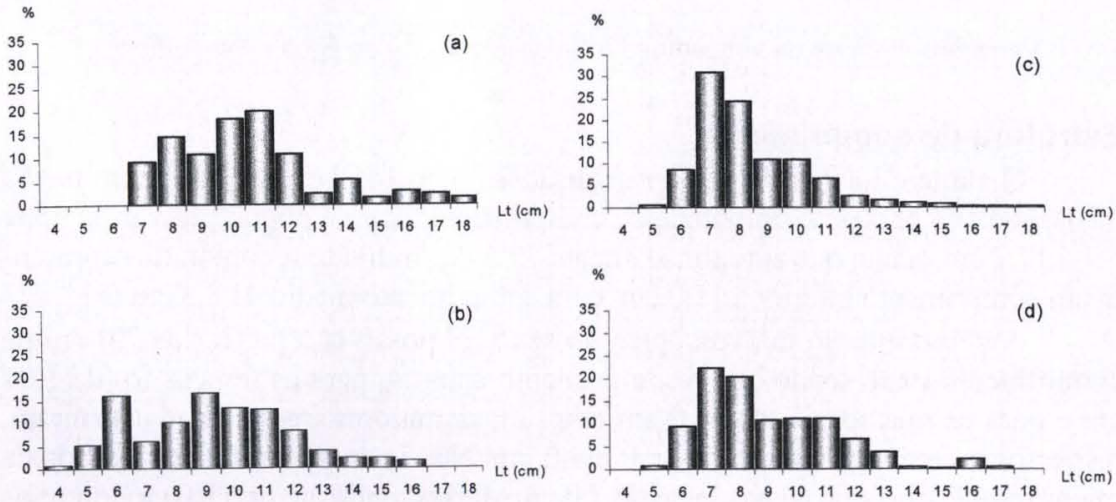

Fig. 3. Distribuição sazonal das classes de comprimento total, primavera (a), verão (b), outono (c) e inverno (d).

coletados, provavelmente pode ser atribuído ao recrutamento dos jovens. A distribuição de comprimento total apresentou padrão bimodal, sendo que as maiores ocorrências foram registradas nas classes de 6,0 e 9,0 cm (Fig. 3b). No outono, os indivíduos com comprimento inferior a $8,0 \mathrm{~cm}$ representaram $64,5 \%$ das capturas, sendo que a moda ocorreu na classe de 7,0 cm (Fig. 3c). Apesar disso, essa estação foi a que apresentou a maior amplitude de comprimento. Nos meses de inverno o padrão de distribuição do comprimento foi semelhante ao outono, com uma maior concentração de exemplares nas classes abaixo de $8,0 \mathrm{~cm}$, apesar de menos evidente que na estação anterior; a moda manteve-se na classe de 7,0 cm (Fig. 3d). 
Tab. I. Freqüência de ocorrência por sexo e exemplares indeterminados de $S$. stellifer, durante o período de julho/1996 a agosto/1997.

\begin{tabular}{|c|c|c|c|c|c|c|}
\hline \multirow[t]{2}{*}{ Mês } & \multicolumn{2}{|c|}{ Fêmeas } & \multicolumn{2}{|c|}{ Machos } & \multicolumn{2}{|c|}{ Indeterminados } \\
\hline & $\mathrm{N}$ & $\%$ & $\mathrm{~N}$ & $\%$ & $N$ & $\%$ relativa \\
\hline Julho/1996 & 34 & 23,61 & 8 & 5,56 & 102 & 70,83 \\
\hline Agosto & 45 & 62,50 & 2 & 2,78 & 25 & 34,72 \\
\hline Setembro & 7 & 87,50 & 1 & 12,50 & - & - \\
\hline Outubro & 17 & 77,27 & 4 & 18,18 & 1 & 4,55 \\
\hline Novembro & 49 & 62,03 & 25 & 31,64 & 5 & 6,33 \\
\hline Dezembro & 20 & 66,67 & 9 & 30,00 & 1 & 3,33 \\
\hline Janeiro/1997 & 56 & 48,70 & 17 & 14,78 & 42 & 36,52 \\
\hline Fevereiro & 94 & 44,34 & 34 & 16,04 & 84 & 39,62 \\
\hline Março & 43 & 60,56 & 16 & 22,54 & 12 & 16,90 \\
\hline Abril & 62 & 26,05 & 17 & 7,14 & 159 & 66,81 \\
\hline Maio & 38 & 16,96 & 17 & 7,60 & 169 & 75,45 \\
\hline Junho & 24 & 18,90 & - & - & 103 & 81,10 \\
\hline Julho & 14 & 30,43 & 6 & 13,04 & 26 & 56,52 \\
\hline Agosto & 21 & 29,58 & 9 & 12,68 & 41 & 57,75 \\
\hline Total & 524 & 35,91 & 165 & 11,31 & 770 & 52,78 \\
\hline
\end{tabular}

\section{Proporção sexual}

Na distribuição da frequiência relativa de machos e fêmeas capturados, durante o período de estudo, as fêmeas dominaram nas amostragens, sendo que o teste do $X^{2}$ indicou diferença significativa $(\mathrm{p}<0,05)$ em todos os meses, exceto em junho/1997, onde não ocorreram machos (Fig. 4). A razão sexual entre machos e fêmeas, ao longo do período estudado foi de 1: 3,2.

Na distribuição da frequiência relativa por classe de comprimento total, as fêmeas contribuíram com as maiores freqüências em todas as classes, sendo observado diferença significativa em todas as classes, exceto na de $9,0 \mathrm{~cm}$; na classe de 18,0 cm não foram registrados machos (Fig. 5).

\section{Relação peso/comprimento}

Os valores da relação $\mathrm{Wt} / \mathrm{Lt}$ foram plotados em gráfico, para sexos agrupados, resultando na equação exponencial Wt $=0,0073 \mathrm{Lt}^{3,1415}$ (Fig. 6a). Linearizando essa relação, verificou-se uma boa aderência dos pontos empíricos à reta, com coeficiente de correlação $r^{2}=0,9819$ (Fig. 6b).

\section{Aspectos reprodutivos}

O tamanho médio de primeira maturação gonadal estimado para as fêmeas foi de 7,5 cm de comprimento total e para os machos de $8,1 \mathrm{~cm}$ (Fig. 7), sendo que o tamanho no qual $100 \%$ dos indivíduos estão aptos a se reproduzirem está em torno de $10,0 \mathrm{~cm}$.

Analisando a população de $S$. stellifer, em função do tamanho de primeira maturação (Fig. 8), verificou-se que a pesca dirigida ao camarão sete-barbas está atuando praticamente de maneira igual sobre os estoques adultos $(51,0 \%)$ e juvenis $(49,0 \%)$.

A análise das variações mensais dos valores médios da relação gonadossomática (RGS) indica que a espécie apresenta dois períodos de desova ao longo do 

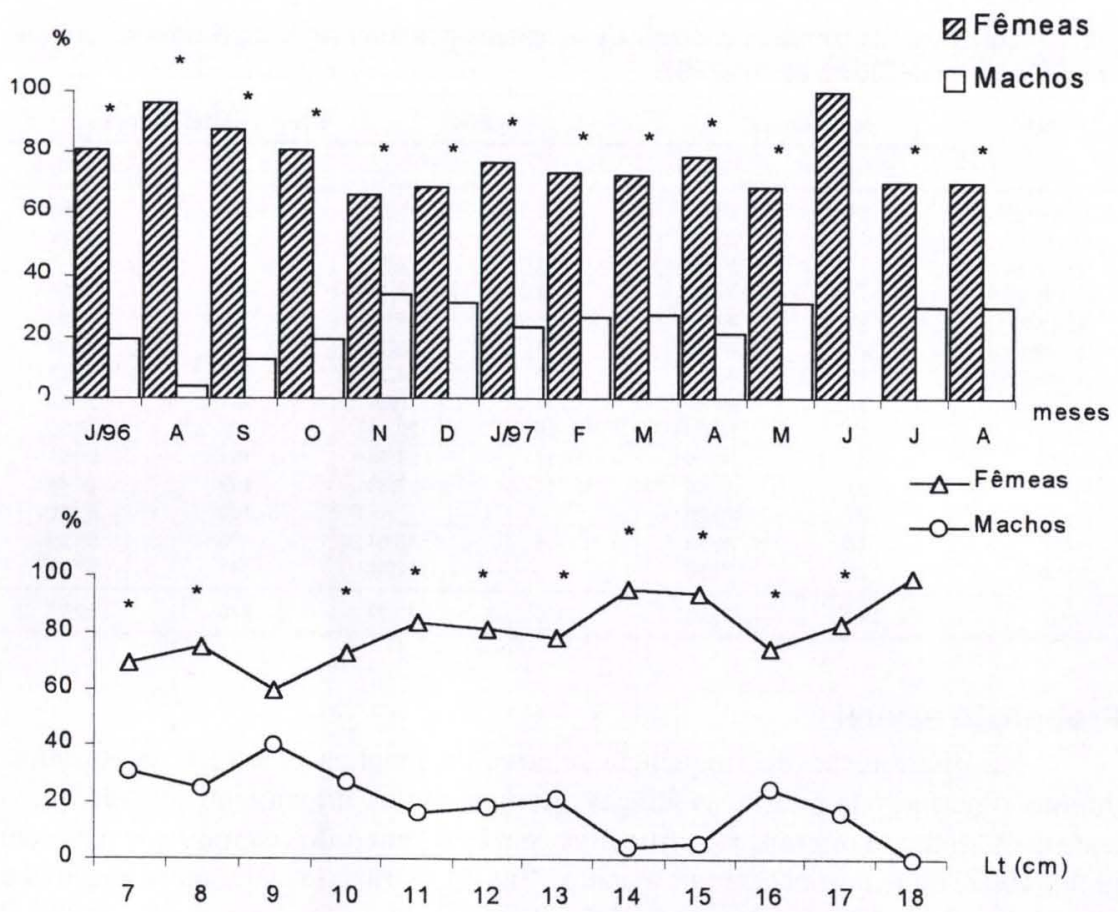

Figs 4-5. Freqüência de ocorrência (\%) por sexos (4) e por classe de comprimento total de machos e fêmeas (5), durante o período de estudo. ( $\left.{ }^{*}\right)$ Indica diferença significativa $X^{2}(p<$ $0,05)$.

(a)

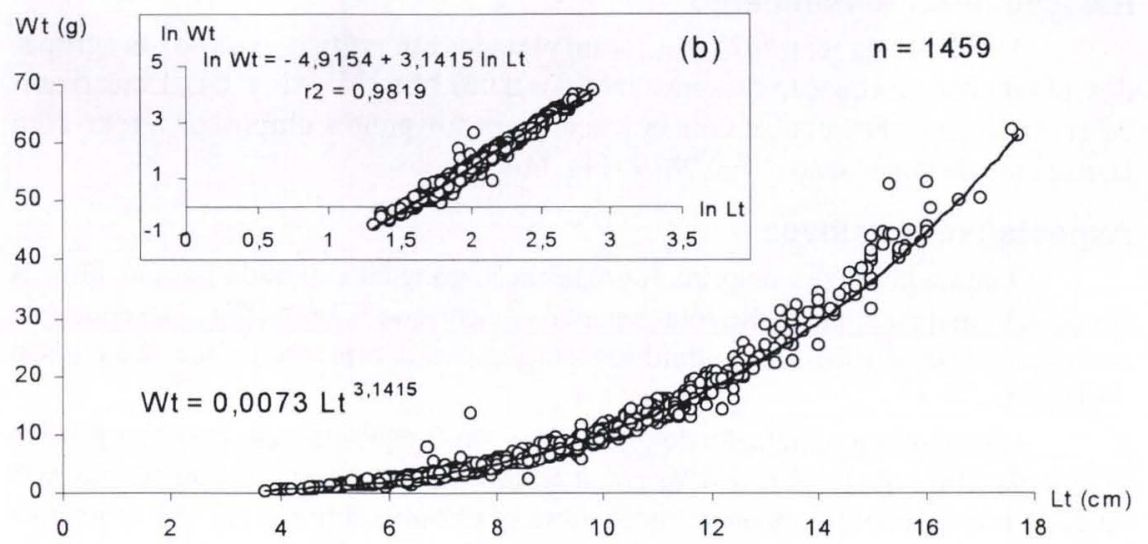

Fig. 6. Relação peso/comprimento (a), e sua transformação logarítmica (b). 


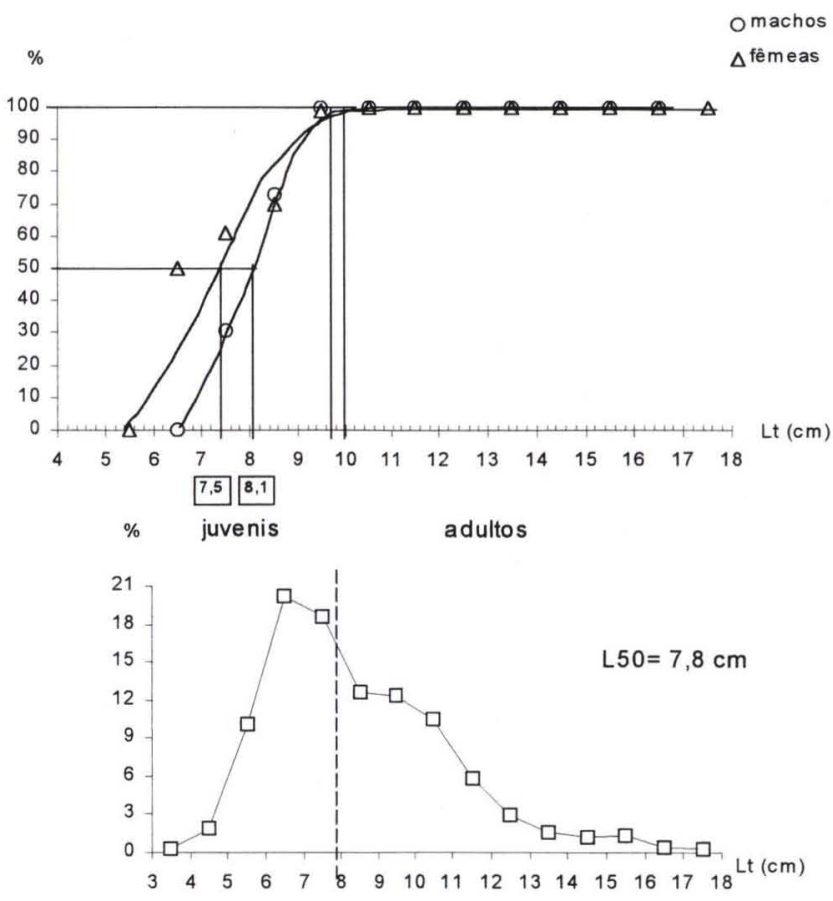

Figs 7-8. Distribuição de freqüência acumulada de machos e fêmeas adultos, por classe de comprimento total $(7)$ e por comprimento, com tamanho de primeira maturação médio $\left(L_{50}\right)$, para sexos agrupados (8). Fêmeas: $L t_{p m}=7,5 \mathrm{~cm}$; machos: $L t_{p m}=8,1 \mathrm{~cm}$.

ano (Fig. 9). Tanto para as fêmeas (Fig. 9a) como para os machos (Fig. 9b), constatou-se valores crescentes a partir de setembro, com o máximo em novembro (fêmeas) e dezembro (machos). Nos meses seguintes os valores decaíram, indicando a desova. Um segundo período reprodutivo, de menor intensidade foi observado a partir de março, seguido de um leve incremento até abril.

\section{DISCUSSÃO}

$\mathrm{Na}$ Armação do Itapocoroy, a temperatura da água de superfície apresentou uma flutuação sazonal, com elevação a partir da primavera até o verão, com posterior queda no outono, até atingir as menores temperaturas no inverno. A variação total da temperatura da água de superfície foi de $11,0^{\circ} \mathrm{C}$ durante o período analisado. A mesma variação sazonal da temperatura da água de superfície foi observada por BRANCO et al. (1999) nas proximidades da área de estudo.

A salinidade da água de superfície variou até $5 \mathrm{ppm}$ durante o período analisado, com oscilações no decorrer do ano. Para a região sul do Brasil, esse padrão não era o esperado, uma vez que MATSUURA (1986) encontrou uma regularidade nas variações da salinidade; apesar disso, essas flutuações observadas na área de estudo são típicas de zonas costeiras, sendo determinadas provavelmente devido a períodos de maior pluviosidade, com grande contribuição fluvial do Rio Itajaí-Açú, 

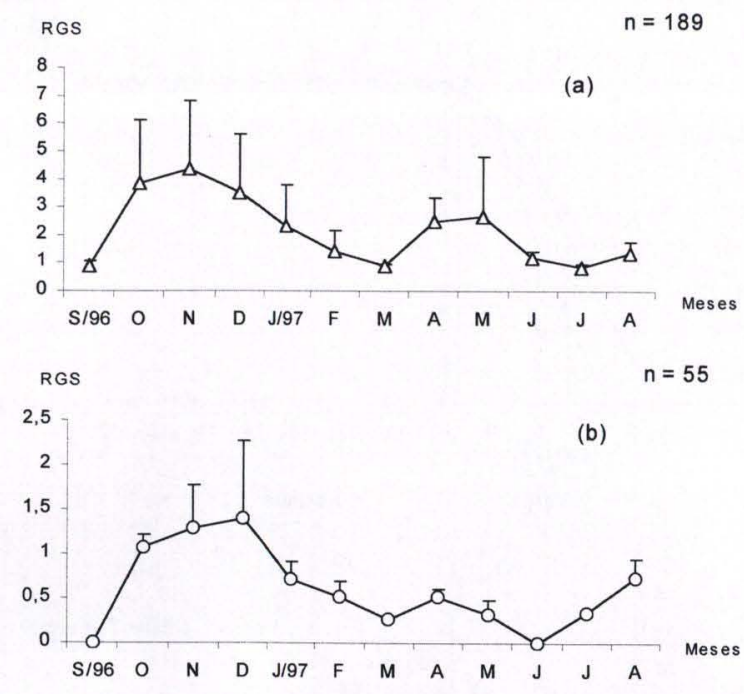

Fig. 9. Relação gonadossomática, por mês, com desvio padrão, para fêmeas (a) e machos (b).

cuja foz está localizada a aproximadamente $20 \mathrm{~km}$ ao sul da Armação do Itapocoroy (BRANCO et al. 1999). O padrão de temperatura na área de coleta esteve de acordo com aquele registrado para a região (MATSUURA 1986).

As capturas de Stellifer stellifer não encontram-se diretamente relacionadas com os parâmetros físico-químicos de água de superfície; as maiores capturas variaram em temperaturas de 19,0 a $27,0^{\circ} \mathrm{C}$ e salinidade de 30,0 a $35,0 \mathrm{ppm}$.

Devido a ausência de informação na literatura especializada sobre $S$. stellifer, os dados foram comparados com os de $S$. brasiliensis e $S$. rastrifer, espécies capturadas como acompanhando a pesca dirigida ao camarão sete-barbas.

A diferenciação sexual macroscópica foi possível somente a partir de exemplares com $6,0 \mathrm{~cm}$ de comprimento total, devido ao reduzido tamanho das gônadas em indivíduos menores, principalmente fora da época de reprodução. Isso acarretou na ocorrência de exemplares indeterminados em grande parte das amostras $(52,8 \%)$. COELHO et al. (1987) também encontraram uma indefinição macroscópica das gônadas dos indivíduos com comprimento total menor que $7,5 \mathrm{~cm} \mathrm{em} S$. brasiliensis, atingindo $36,2 \%$ dos exemplares capturados.

Quanto à distribuição da frequência de ocorrência do comprimento total em relação às estações do ano, pode-se verificar que a entrada dos recrutas está restrita aos meses de verão. $\mathrm{Na}$ primavera foram registrados os maiores exemplares da população, o que coincide com os maiores valores da relação gonadossomática.

A proporção entre fêmeas e machos é uma informação importante para caracterização da estrutura de uma espécie ou população, além de fornecer subsídio para o estudo de outros aspectos bioecológico como a avaliação do potencial reprodutivo e estimativas do tamanho do estoque (VAZZOLER 1996). 
S. stellifer apresentou uma dominância de fêmeas em todos os meses do ano e de classes de comprimento. Segundo CoELHO et al. (1987) o predomínio de fêmeas ao longo do ano, na pesca artesanal do camarão sete-barbas, acarreta em maior dano a população, pois a abundância das fêmeas é um dos principais fatores que depende o potencial reprodutivo de uma população.

A relação peso/comprimento tem sido utilizada para facilitar a estimativa do peso de um exemplar através de seu comprimento, sendo amplamente empregada em estudos de dinâmica populacional e avaliação de estoques pesqueiros (BRANCO et al. 1999). A importância dessa relação está em conhecer a forma de crescimento de uma espécie, pois populações distintas de uma mesma espécie podem apresentar taxas diferentes de crescimento (VAZZOLER 1996).

A relação peso/comprimento ajustada para Stellifer stellifer, apresentou tendência exponencial, sendo o padrão de crescimento do tipo alométrico positivo. O mesmo padrão de crescimento foi encontrado para $S$. brasiliensis e $S$. rastrifer (GIANINNI \& PAIVA-FILHO 1990, 1995; COELHO et al. 1985; CHAveS \& VENDEL 1997).

A estimativa do tamanho de primeira maturação é fundamental para a administração racional dos estoques, sendo que a sobreposição desse tamanho com as curvas de distribuição de frequência por comprimento permitem determinar o estrato da população em que a pesca vem atuando com maior intensidade (BRANCO et al. 1999). Quanto a atuação da pesca sobre a população de S. stellifer, na Armação do Itapocoroy, observou-se que a quantidade de juvenis e adultos capturados foi muito semelhante.

A relação gonadossomática mostrou-se adequada como um estimador da época de desova de $S$. stellifer, indicando a ocorrência de dois períodos de desova, um mais marcado na primavera, se estendendo até o verão, e um menos intenso no outono.

\section{REFERÊNCIAS BIBLIOGRÁFICAS}

Branco, J.O; M.J. Lunardon-Branco; F.X. Souto \& C.R. Guerra. 1999. Estrutura populacional do camarão sete-barbas Xiphopenaeus kroyeri (Heller, 1862), na foz do rio Itajaí-Açú, Itajaí, SC, Brasil. Braz. arch. biol. technol., Curitiba, 42 (1): 115-126.

Chaves, P. DE T. DA C. \& A.L. Vendel. 1997. Reprodução de Stellifer rastrifer (Jordan) (Teleostei, Sciaenidae) na baía de Guaratuba, Paraná, Brasil. Revta bras. Zool. 14 (1): 81-89.

1998. Feeding habits of Stellifer rastrifer (Perciformes, Sciaenidae) at Guanabara Mangrove, Paraná, Brazil. Braz. arch. biol. technol., Curitiba, 41 (4): 423-428.

Coelho, J.A.P.; R. GraçA-Lopes; E.S. Rodrigues \& A. Puzzi. 1985. Relação peso-comprimento e tamanho de início de primeira maturação gonadal para o Sciaenidae Stellifer rastrifer (Jordan, 1889), no litoral do Estado de São Paulo. Bol. Inst. Pesca, São Paulo, 12 (2): 99-107.

Coelho, J.A.P.; A. Puzzi; R. GraçA-Lopes; E.S. Rodrigues \& J.R.O. Preto. 1986. Análise da rejeição de peixes na pesca artesanal dirigida ao camarão sete-barbas (Xiphopenaeus kroyeri) no litoral do Estado de São Paulo. Bol. Inst. Pesca, São Paulo, 13 (2): 51-61.

Coelho, J.A.P.; R. GraÇA-Lopes; E.S. Rodrigues \& A. Puzzi. 1987. Aspectos biológicos e pesqueiros do Sciaenidae Stellifer brasiliensis (Schultz, 1945), presente na pesca artesanal dirigida ao camarão sete-barbas (São Paulo, Brasil). Bol. Inst. Pesca, São Paulo, 14: 1-10.

Gianinni, R. \& A.M. Paiva-Filho. 1990. Aspectos bioecológicos de Stellifer rastrifer (Perciformes: Sciaenidae) na Baía de Santos, SP. Bol. Inst. Pesca, São Paulo, 38 (1): 57-67.

Revta bras. Zool. 19 (2): 601 - 610, 2002 
1995. Distribuição temporal, espacial e bioecologia do cangoá, Stellifer brasiliensis (Teleostei: Sciaenidae), na Baía de Santos, São Paulo, Brasil. Arq. Ciên. Mar, Fortaleza, 29 (1-2): 5-15.

Lopes, F.R.A.; A. CAFFE; M. HostiM-Silva; V. INEZ \& L. MACHADO. 1997. Abundância e distribuição espaço-temporal de Stellifer rastrifer (Perciformes - Sciaenidae) na foz do rio Itajaí-Açu, SC, Brasil. In: Anais Semana Nacional de Oceanografia, Itajaí, FACIMAR/UNIVALI, p. 364-366.

MAtsuURA, Y. 1986. Contribuição ao estudo da estrutura oceanográfica da região Sudeste entre Cabo Frio (RJ) e Cabo de Santa Marta (SC). Ci. Cult. 38 (8): 1439-1450.

Menezes, N.A. \& J.L. Figueiredo. 1980. Manual de peixes marinhos do sudeste do Brasil: IV Teleostei (3). São Paulo, Museu de Zoologia da Universidade de São Paulo, 96p.

SANTOS, E.P. DOS. 1978. Dinâmica de populações aplicada à pesca e piscicultura. São Paulo, Hucitec, USP, 129p.

VAZZOLER, A.E.A.M. 1996. Biologia da reprodução de peixes teleósteos: teoria e prática. Co-edição EDUEM/CNPq e Nupelia, 169p.

Recebido em 03.VIII.2001; aceito em 22.V.2002. 